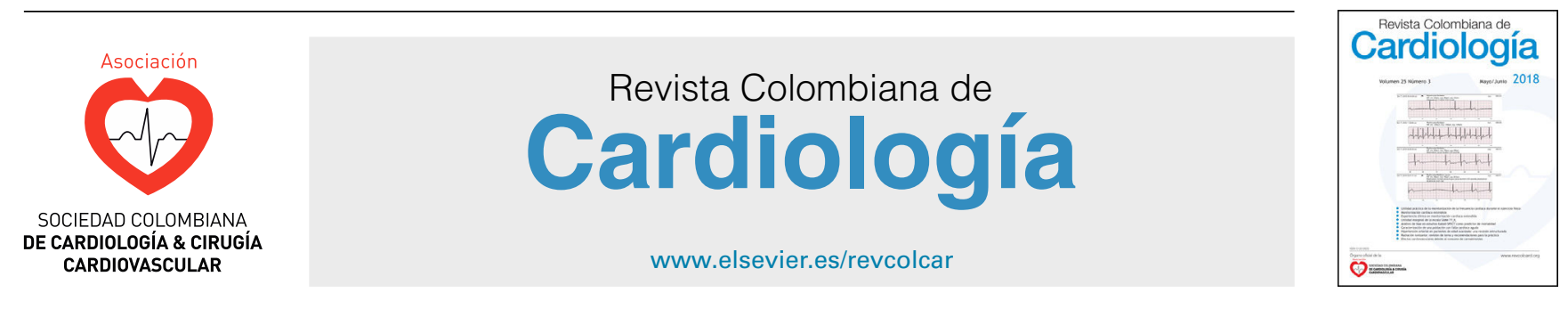

CARDIOLOGÍA DEL ADULTO - PRESENTACIÓN DE CASOS

\title{
Caso clínico de cor triatriatum en el adulto asintomático: diagnóstico ecocardiográfico
}

\author{
Eliza Kaori Uenishi *, Clara I. Arias-Barrera, Liliana Pitre-Velásquez \\ y Carlos A. Ariza-La Rotta
}

\begin{abstract}
Fundación Cardiovascular de Colombia- Instituto del Corazón Santa Marta, Santa Marta, Colombia
\end{abstract}
Recibido el 19 de octubre de 2017; aceptado el 6 de septiembre de 2019

Disponible en Internet el 27 de abril de 2020

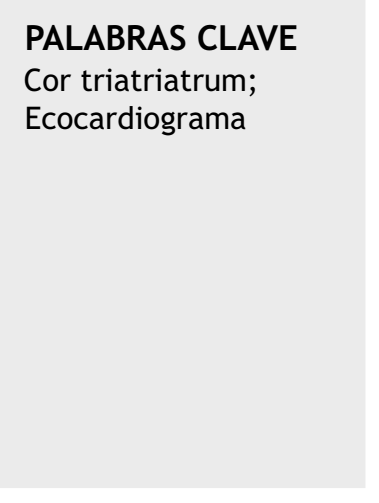

KEYWORDS

Cor triatriatum;

Cardiac ultrasound

\begin{abstract}
Resumen El cor triatriatum es una anomalía congénita rara, que representa el $0,4 \%$ de todas las malformaciones cardíacas. En esta malformación la aurícula es separada en dos compartimientos por una membrana fibromuscular, que puede ser enteramente intacta o contener fenestraciones de diámetros variables. La aparición de la sintomatología está en relación con las propiedades obstructivas que mimetizan la estenosis mitral y la presencia de una comunicación interauricular. La ecocardiografía bidimensional y el Doppler color son las modalidades diagnósticas no invasivas de elección para el diagnóstico preciso del cor triatriatum y de su repercusión hemodinámica. Se reporta un caso de cor triatriatum en un paciente adulto mayor, en quien se hizo el diagnóstico tardío y casual de esta malformación.

(c) 2019 Sociedad Colombiana de Cardiología y Cirugía Cardiovascular. Publicado por Elsevier España, S.L.U. Este es un artículo Open Access bajo la licencia CC BY-NC-ND (http:// creativecommons.org/licenses/by-nc-nd/4.0/).
\end{abstract}

\section{Clinical case of cor triatriatum in the asymptomatic adult: cardiac ultrasound diagnosis}
Abstract Cor triatriatum is a rare congenital anomaly that represents $0.4 \%$ of all cardiac malformations. In this malformation the atrium is separated into two compartments by a fibrous-muscular membrane that may be completely intact or contain fenestrations with varia- ble diameters. The appearance of symptoms is associated with obstruction-like properties that mimic mitral stenosis and an atrial septal defect. Two-dimensional cardiac ultrasound and colour Doppler are non-invasive methods of choice for the accurate diagnosis of cor triatriatum and its haemodynamic repercussions. A case of cor triatriatum is reported in an elderly patient, in whom a delayed and casual diagnosis was made of this malformation.
(c) 2019 Sociedad Colombiana de Cardiología y Cirugía Cardiovascular. Published by Else- vier España, S.L.U. This is an open access article under the CC BY-NC-ND license (http:// creativecommons.org/licenses/by-nc-nd/4.0/).

* Autor para correspondencia.

Correo electrónico: ezkaori@yahoo.com.br (E.K. Uenishi). 


\section{Introducción}

El cor triatriatum es una anomalía congénita rara, que generalmente se diagnostica durante la niñez. Fue descrito por primera vez en 1868 por Church $^{1}$, representa el $0,4 \%$ de todas las malformaciones cardíacas ${ }^{2}$ y se encuentra en menos del $0,1 \%$ de las cardiopatías clínicamente diagnosticadas ${ }^{3}$.

En cuanto a embriología, el cor triatriatum sinistrum se interpreta como el resultado de una incorporación incompleta de la vena pulmonar común embrionaria a la aurícula izquierda, que forma como resultado una cámara auricular proximal o accesoria a la aurícula verdadera, separada en dos compartimientos por una membrana fibromuscular. La membrana que separa el atrio en dos partes varía significativamente en tamaño y forma. Puede ser enteramente intacta o contener uno o más orificios (fenestraciones) de diámetros variables. De acuerdo con el grado de integridad de la membrana el cor triatriatum puede ser clasificado como obstructivo o no obstructivo. Los casos no obstructivos pueden ser asintomáticos y su diagnóstico en adultos se realiza de manera casual tras el estudio ecocardiográfico por otras indicaciones. La aparición de los síntomas está en relación con las propiedades obstructivas que mimetizan la estenosis mitral y la presencia de una comunicación interauricular. El área de apertura de la membrana no se altera con la edad y la presentación tardía puede obedecer al desarrollo de complicaciones, como insuficiencia mitral o fibrilación auricular. A pesar de no haber signos o síntomas típicos que distingan el cor triatriatum de otras entidades, la disnea es el síntoma más identificado en adultos. Las arritmias son principalmente supraventriculares y la insuficiencia mitral suele ser el desencadenante de cuadros sintomáticos en los pacientes ${ }^{4}$. Tanto el momento de aparición de los síntomas como el pronóstico de estos pacientes, dependen de dos factores: el diámetro de perforación del orificio de la membrana divisoria fibromuscular y, por otro lado, el grado de descompresión del compartimiento superior a través de una comunicación interauricular, o al conectarse ésta a una vía venosa pulmonar alterna.

El objetivo de este reporte es exponer el caso de un paciente adulto mayor con una cardiopatía congénita de rara presentación, en quien se diagnosticó de manera tardía y ocasional cor triatriatum sinistrum típico.

\section{Caso}

Paciente masculino de 76 años de edad, alcohólico, extabaquista severo de medio paquete al día por más de cincuenta años, hasta hace seis meses; antecedente personal de hipertensión arterial diagnosticada hace cinco años en manejo con losartán, amlodipino e hidroclorotiazida; dislipidemia en manejo con atorvastatina; cirugías previas de herniorrafia discal hace 15 años y apendicectomía hace 37 años.

Fue referido a nuestra institución para la realización de exámenes para el rastreo de enfermedad coronaria posterior a consulta externa con cardiología por dolor precordial tipo opresión asociado, a falta de aire de treinta minutos de duración, relacionado con el esfuerzo físico moderado desde hace seis meses (aumento de intensidad y duración progresivamente); últimamente presenta estos síntomas durante el reposo. Refería cuadro clínico de tres días de evolución caracterizado por dolor precordial, punzante, en aumento, acompañado de disnea de medianos esfuerzos y malestar general.

Al examen físico, ruidos cardíacos rítmicos, sin soplo; pulmones con murmullo vesicular disminuido de forma difusa y severa.

El electrocardiograma mostró ritmo sinusal, con isquemia subendocárdica anterior (fig. 1).

La prueba de esfuerzo fue positiva para isquemia miocárdica.

En la ecocardiografía transtorácica se diagnosticó de cor triatriatum. Presencia de dilatación moderada de la aurícula izquierda (volumen indexada $=38 \mathrm{ml} / \mathrm{m}^{2}$ ), dividida en dos cámaras por una membrana perforada, con gran orificio de $23 \mathrm{~mm}$ que comunica ambas cavidades. En la cámara posterior desembocan las venas pulmonares. La cámara anterior contiene la auriculilla y se comunica con el ventrículo izquierdo a través de la válvula mitral, la cual presenta calcificación leve del anillo sin disfunción. No hay gradiente transmembrana intrauricular (fig. 2). Ventrículo izquierdo con función sistólica y contractilidad segmentaria normal. La fracción de eyección del ventrículo izquierdo se estimó en $88 \%$. Septum interauricular íntegro.

El Holter cardíaco de $\mathbf{2 4}$ horas mostró extrasístoles ventriculares monomórficas aisladas ocasionales, extrasístoles auriculares conducidas ocasionales y evento de taquicardia auricular no sostenida.

El cateterismo cardíaco izquierdo y derecho reportó lesiones graves en tres coronarias (descendente anterior, circunfleja y coronaria derecha). Presión de ventrículo izquierdo: 140/12 mm Hg. Presión de aorta 140/80 mm Hg. No existe gradiente de presión entre aorta y ventrículo izquierdo. Arteriografía pulmonar normal, buen retorno venoso a aurícula izquierda, presión de arteria pulmonar: $32 / 9 \mathrm{~mm} \mathrm{Hg}$, con una media de $14 \mathrm{~mm} \mathrm{Hg}$, presión en cuña de arteria pulmonar $9 \mathrm{~mm} \mathrm{Hg}$ (fig. 3). En el cateterismo derecho no reportó comunicación interauricular u otras malformaciones congénitas asociadas.

No le fue realizado ecocardiograma transesofágico debido a la falta de autorización de la entidad prestadora de salud a la que está afiliado el paciente.

Fue sometido a tratamiento quirúrgico de revascularización miocárdica de tres vasos en otra institución, sin presentar ninguna complicación.

\section{Discusión}

En países en desarrollo, como Colombia, el diagnóstico precoz y el tratamiento adecuado de las cardiopatías congénitas no alcanza a llegar al 40\%. Es relativamente frecuente el hallazgo ocasional de cardiopatías congénitas en adultos con síntomas inespecíficos, como disnea, edema de extremidades o dolor en torácico. El aumento en el número de diagnósticos de cardiopatías congénitas en adultos con hallazgo ocasional se debe a la mayor oferta de servicios oportunos realizados por personal más especializado.

La prevalencia de pacientes con cardiopatías congénitas en Colombia es de $9 / 1.000$ nacidos vivos ${ }^{5,6}$. De acuerdo con Sandoval ${ }^{7}$, más del $60 \%$ de los niños no tienen la oportunidad de recibir tratamiento en el primer año de vida. 


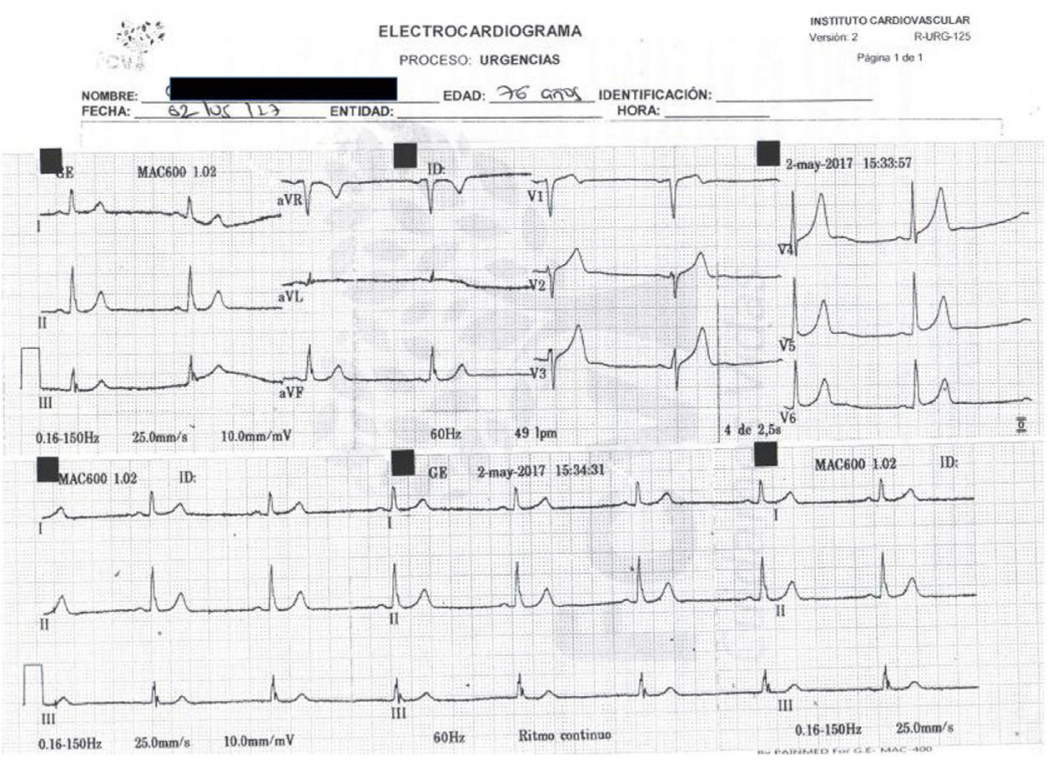

Figura 1 Electrocardiograma de 12 derivaciones.

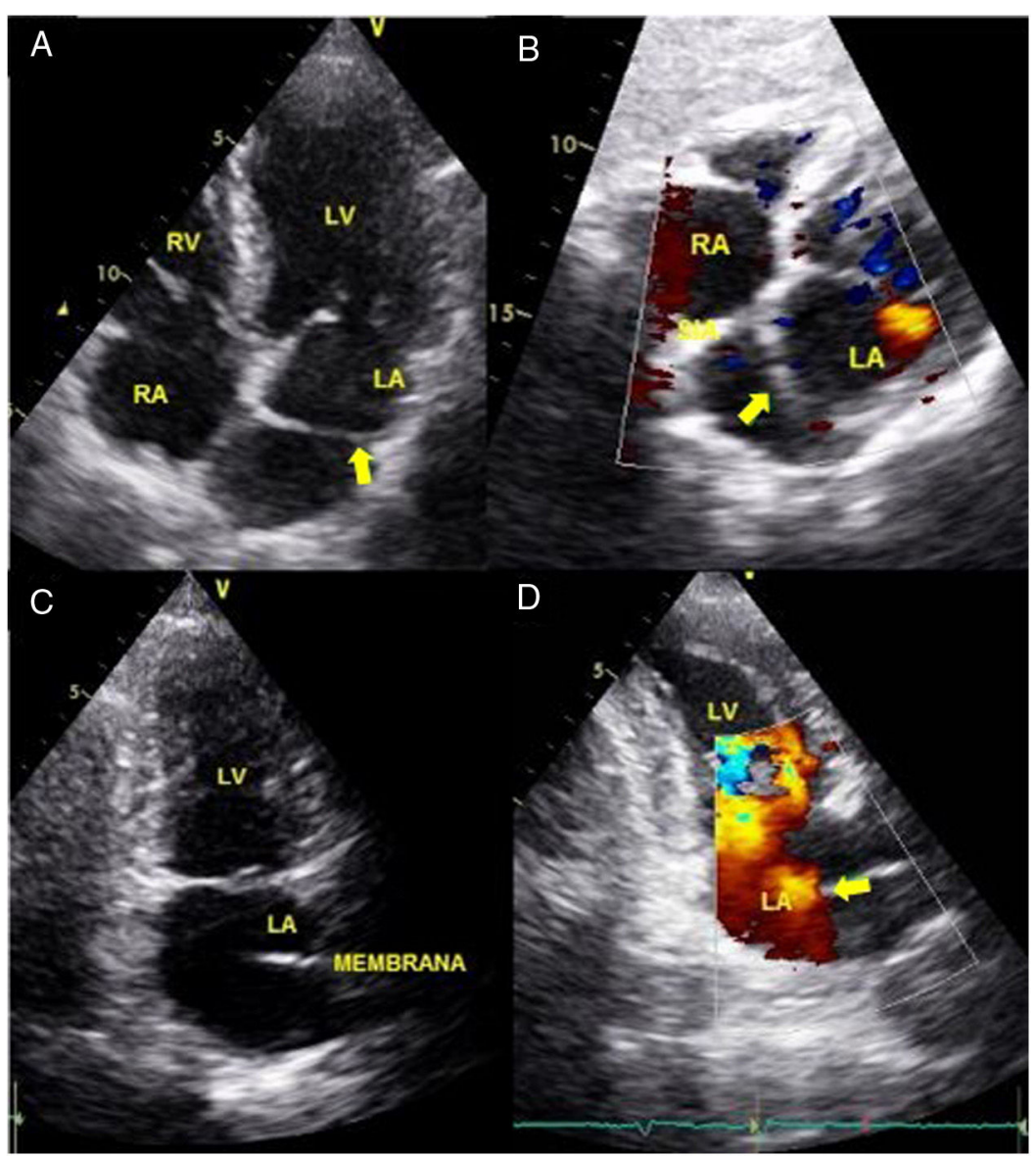

Figura 2 Ecocardiograma transtorácico bidimensional. A. Corte apical 4 cámaras que muestra aurícula izquierda divida en dos compartimientos, separados por una membrana (flecha), proximal y distal. (B) Ventana subcostal que muestra septum interauricular íntegro y una membrana a nivel del foramen oval separando la aurícula izquierda en dos compartimientos. C. Corte apical 2 cámaras que muestra aurícula izquierda dividida por una membrana discontinua (flecha) sin producir gradiente entre las dos cámaras. $\mathrm{D}$. RV: ventrículo derecho; RA: aurícula derecha; LV: ventrículo izquierdo; LA: aurícula izquierda. 


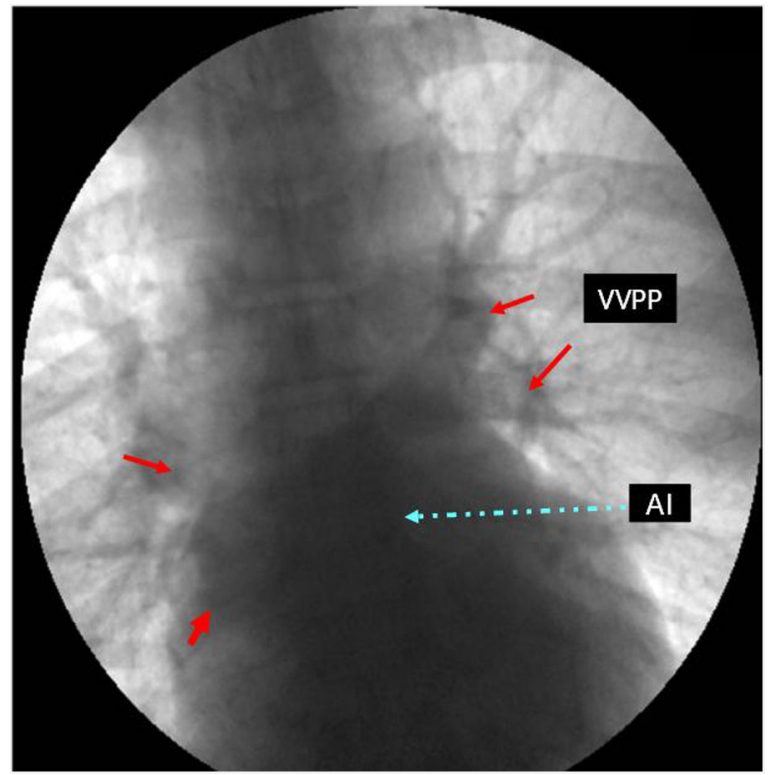

Figura 3 Cateterismo cardíaco que muestra el retorno venoso a la aurícula izquierda sin que se observen estructuras internas definidas tipo membrana.

VVPP: venas pulmonares; Al: aurícula izquierda.

El porcentaje de pacientes con cardiopatías congénitas que recibe tratamiento y sobreviven ha aumentado considerablemente en el mundo, y también en Colombia, lo que contribuye a que un mayor número de estos pacientes lleguen a la edad adulta. Otra parte de la población, probablemente con cardiopatías congénitas menos complejas, llegará a la adultez sin manejo, algunos de los cuales son tratados o no sometidos a procedimientos en esta fase. En este grupo de población adulta hay casos de cardiopatías congénitas diagnosticadas por primera vez, algunas veces como hallazgo ocasional, otras por sintomatologías inespecíficas y que buscan ayuda por manifestaciones de insuficiencia cardíaca. No hay datos estadísticos claros de este segmento de población con cardiopatías congénitas diagnosticadas por primera vez a esta edad. Debido a los avances en la atención y al uso generalizado de técnicas de imágenes en la evaluación de cardiopatías ha habido un aumento en la detección precoz y en la sobrevivencia de los pacientes operados por cardiopatías congénitas durante las últimas décadas ${ }^{8}$.

A pesar del aumento en la sobrevida de personas con cardiopatías congénitas en la edad adulta, se reporta de mayor porcentaje de depresión en estos pacientes, cuando se comparan con la población general, debido a diferentes factores ${ }^{9}$.

Para el establecimiento de diagnóstico de cor triatriatum sinistrum no son necesarios exámenes invasivos. Desde la década de los años ochenta hasta la actualidad, la ecocardiografía bidimensional y el Doppler color se han convertido en la modalidad diagnóstica no invasiva de elección para el diagnóstico preciso del cor triatriatum y de su repercusión hemodinámica, permitiendo valorar la existencia de una membrana fibromuscular y el número de fenestraciones y su tamaño, así como identificar anomalías cardíacas congénitas asociadas y el gradiente transmembrana ${ }^{10}$. La ecocardiografía transesofágica, tridimensional y la resonancia magnética ayudan también a complementar el diagnóstico. El cateterismo cardíaco se reserva para los casos dudosos o ante la sospecha de otras anomalías cardíacas asociadas ${ }^{11}$.

El cor triatriatum es una anomalía congénita rara, aún más en edad adulta, y aislada sin otras cardiopatías congénitas asociadas. En un reporte de la Clínica Mayo ${ }^{12}$, al analizar cincuenta años de cirugía de cor triatriatum sinistrum, hubo 25 casos en este periodo, 21 (84\%) estaban asociados a otras cardiopatías (congénitas o adquiridas). $\mathrm{El}$ paciente que se reporta en este artículo acudió a nuestra institución para la realización de exámenes diagnósticos de síndrome coronario. Fue sometido posteriormente a cirugía de revascularización miocárdica sin la necesidad de complementación de otras correcciones, por no presentar fenómenos obstructivos al flujo.

Los pacientes con cor triatriatum no obstructivo y asintomáticos pueden ser seguidos clínicamente con orientación para la modificación de estilos de vida y control de factores de riesgo cardíaco. Sin embargo, hay que realizar control rutinario con ecocardiografía, dado que, con el tiempo, puede haber calcificación progresiva y fibrosis de la membrana, con consecuente obstrucción del flujo ${ }^{13}$.

\section{Conclusión}

El cor triatriatum sinistrum es una anomalía congénita rara y aún más en pacientes adultos. La forma aislada sin anormalidad cardíaca asociada es más común en la adultez. El ecocardiograma transtorácico es el examen diagnóstico de primera línea de elección para el diagnóstico. El caso reportado aquí es un hallazgo ocasional que, de no haberse manifestado el síndrome coronario, tal vez habría pasado inadvertido.

\section{Conflicto de intereses}

Ninguno.

\section{Agradecimiento}

Al Doctor Oscar Giovanny Iglesias-Jiménez por la colaboración en la traducción al inglés.

\section{Bibliografía}

1. Church WS. Congenital malformations of the heart: Abnormal septum in the left auricle. Trans Pathol Soc Lond. 1868;19:188-90.

2. Jegier W, Gibbons JE, Wiglesworth FW. Cor triatriatum: clinical, hemodynamic and pathological studies surgical correction in early life. Pediatrics. 1963;31:255-67.

3. Talner CN. Report of the New England Regional Infant Cardiac Program, by Donald C. Fyler, MD. Pediatrics. 1998;102(1 pt 2):258-9.

4. Geggel RL, Fulton DR, Rockenmancher S. Nonobstructive cor triatriatum in infancy. Clin Pediatrics. 1999;38:489-91.

5. Van der Linde D. Birth prevalence of congenital heart disease worldwide. A Systematic review and meta-analysis. J Am Coll Cardiol. 2011;58:2241-7.

6. Zarante I. Frecuencia de malformaciones congénitas, evaluación y pronóstico de 52.744 nacimientos de tres ciudades colombianas. Medica. 2010;30:65-71. 
7. Sandoval N. Adultos con cardiopatías congénitas: una población creciente Retos del presente y el futuro. Rev Colomb Cardiol. 2017;24:199-203.

8. Gilboa S, Devine OJ, Kucik JE, Oster ME, Riehle-Colarusso T, Nembhard WN, et al. Congenital heart defects in the United States Estimating the magnitude of the affected population 2010. Circulation. 2016;134:101-9.

9. Pauliks LB. Depression in adults with congenital heart disease-public health challenge in a rapidly expanding new patient population. World J Cardiol. 2013;5: 186-95.
10. Alphonso N, Norgaard MA, Newcomb A, d'Udekem Y, Brizard C, Cochrane A. Cor triatriatum: Presentation, diagnosis and longterm surgical results. Ann Thorac Surg. 2005;80:1666-71.

11. Nassar PN, Hamdan RH. Cor triatriatum sinistrum: classification and imaging modalities. Eur J Cardiovasc Med. 2011;1:84-7.

12. Saxena P, Burkhart HM, Schaff HV, Daly R, Joyce LD, Dearani JA. Surgical repair of cor triatriatum sinister: the mayo clinic 50-year experience. Ann Thorac Surg. 2014;97:1659-63.

13. Zepeda IA, Morcos P, Castellanos LR. Cor triatriatum sinister identified after new onset atrial fibrillation in an elderly man. Case Rep Med. 2014, http://dx.doi.org/10.1155/2014/674018. 